\title{
Pulmonary alveolar microlithiasis: blizzard of lung
}

\author{
Richa Mittal, Rohit Kumar, Juvva Kishan Srikanth, Siddharth Raj Yadav, Ravi Chandak, \\ Shibdas Chakrabarti
}

Department of Pulmonary, Critical Care and Sleep Medicine, Vardhman Mahavir Medical College and Safdarjung Hospital, New Delhi, India

\begin{abstract}
Pulmonary alveolar microlithiasis (PAM) is a rare inherited disorder in which patients present with either nonspecific signs and symptoms or are asymptomatic. Clinical-radiological dissociation is a commonly seen feature of the disease. The majority of cases are diagnosed in second and third decade of life, although presentation in paediatric and elderly population is also reported. Pathognomonic radiological findings, with normal calcium and phosphate levels can confirm the diagnosis without the need of further investigations in large number of cases. A high index of suspicion and knowledge of the disease is of paramount importance as it has a wide range of presentation and variable
\end{abstract}

Correspondence: Rohit Kumar, Department of Pulmonary Medicine, Critical Care Medicine and Sleep Medicine, Vardhman Mahavir Medical College and Safdarjung Hospital, New Delhi, India. Tel. +919911218081.

E-mail: dr.rohitkumar@mail.com

Author's contributions: RM, RK and SC contributed to the design of the report. RM, RK, JKS, SRY, RC and SC contributed to the discussion and review of the topic. RM, JKS and SRY drafted the manuscript. RM, RK, JKS, SRY and SC critically revised the manuscript. All the authors gave final approval and agree to be accountable for all aspects of the report ensuring integrity and accuracy.

Conflict of interest: The authors declare no potential conflict of interest.

Informed consent: Written informed consent was taken from both the patients.

Availability of data and materials: All data generated or analyzed during this study are included in this published article.

Keywords: Pulmonary alveolar microlithiasis; microliths; pulmonary calcifications.

Received for publication: 1 December 2020.

Accepted for publication: 22 April 2021.

${ }^{\circ}$ Copyright: the Author(s), 2021

Licensee PAGEPress, Italy

Monaldi Archives for Chest Disease 2021; 91:1709

doi: 10.4081/monaldi.2021.1709

This article is distributed under the terms of the Creative Commons Attribution Noncommercial License (by-nc 4.0) which permits any noncommercial use, distribution, and reproduction in any medium, provided the original author(s) and source are credited. course thus posing a diagnostic challenge. We present two cases of this rare disease in elderly females with distinct presentations although with similar and characteristic radiological findings along with a brief review of literature of the disease.

\section{Introduction}

Pulmonary alveolar microlithiasis (PAM) is an orphan disease, characterised by intra alveolar deposition of calcium phosphate microliths. It is a rare genetic disorder with highest prevalence in Asian population [1]. PAM was first introduced by Marcello Malpighi in 1868 but was acknowledged and described as microlithiasis alveolaris pulmonum only in 1933 by a Hungarian pathologist, Puhr [2]. The first case of PAM in India was reported by Viswanathan et al. in 1961 [3]. Though diagnosed chiefly between the ages of 20-30 years, presentation among children and elderly have also been described. Dissociation of severity of symptoms and radiological findings is an essential distinguishing feature of the disease. The majority of patients are diagnosed incidentally and are asymptomatic at presentation. It has a variable course of illness with poor longterm survival [1]. The best diagnostic work-up consists of chest radiography followed by combination of high-resolution computed tomography (HRCT) of the chest with bronchoalveolar lavage (BAL).

We present two cases of this rare disease in elderly females with contrasting presentations.

\section{Case \#1}

A 56-year-old Indian female, never smoker, housewife, with no comorbidities presented to the outpatient department of a tertiary care hospital with the chief complaints of dry cough and breathlessness on exertion which progressed from modified Medical Research Council (mMRC) grade I to grade III over a period of 3 years. The patient denied any history of fever, chest pain, significant loss of weight or appetite and there was no history of exposure to biomass fuel or silica dust. Her past medical history and family history were unremarkable. Patient was prescribed inhaled bronchodilators, which she used to take on and off and there was only partial relief after using the same.

On physical examination the patient had a pulse rate of $98 / \mathrm{min}$, respiratory rate of $22 / \mathrm{min}$, no pedal edema and pan digital clubbing of grade 3 was observed. Respiratory system examination revealed bilateral fine, end inspiratory crackles in all areas of lung. Baseline investigations showed haemoglobin of $14.7 \mathrm{~g} / \mathrm{dl}$, 
haematocrit of 42.8 and a total leucocyte count of $9200 / \mathrm{mm}^{3}$. Resting arterial blood gas values on room air revealed $\mathrm{pH} 7.46$, $\mathrm{pCO}_{2} 34 \mathrm{~mm} \mathrm{Hg}, \mathrm{pO}_{2} 62 \mathrm{~mm} \mathrm{Hg}, \mathrm{HCO}_{3} 21 \mathrm{mmol} / \mathrm{l}$ and oxygen saturation of $93 \%$. Pulmonary function tests (PFT) demonstrated moderate restrictive defect with severe reduction in diffusion capacity: forced expiratory volume in $1 \mathrm{~s}\left(\mathrm{FEV}_{1}\right) 65 \%$, forced vital capacity (FVC) 64\%, FEV1/FVC 88\%, total lung capacity (TLC) $66 \%$ and diffusion lung capacity (DLCO) $36 \%$. The patient's sixminute walk test (6MWT) distance was 254 metres with significant desaturation (up to $80 \%$ ).

Her chest X-ray (CXR) showed bilateral dense, nodular opacities throughout the lung parenchyma, predominantly in the middle and lower zones (Figure 1a). There were no previous X-rays or CT scans available for comparison. HRCT chest was performed, and it showed multiple sand like nodules of calcium density with alveolar distribution in all lobes (distinctly visualised in the mediastinal window), and interlobular septal thickening and predominance in the pulmonary bases. There were small sub pleural cysts and a characteristic black pleura sign was seen (Figure 2a). The nodules were more confluent in the inferior regions of lung (Figure $3 a$ ). No significant lymphadenopathy or pleural or pericardial effusion was observed.

Induced sputum examination was negative for acid fast bacilli (AFB) or gram stain. Echocardiogram reported evidence of moderate pulmonary hypertension with mean pulmonary artery pressure of $34 \mathrm{~mm} \mathrm{Hg}$, mild dilatation of right ventricle and left
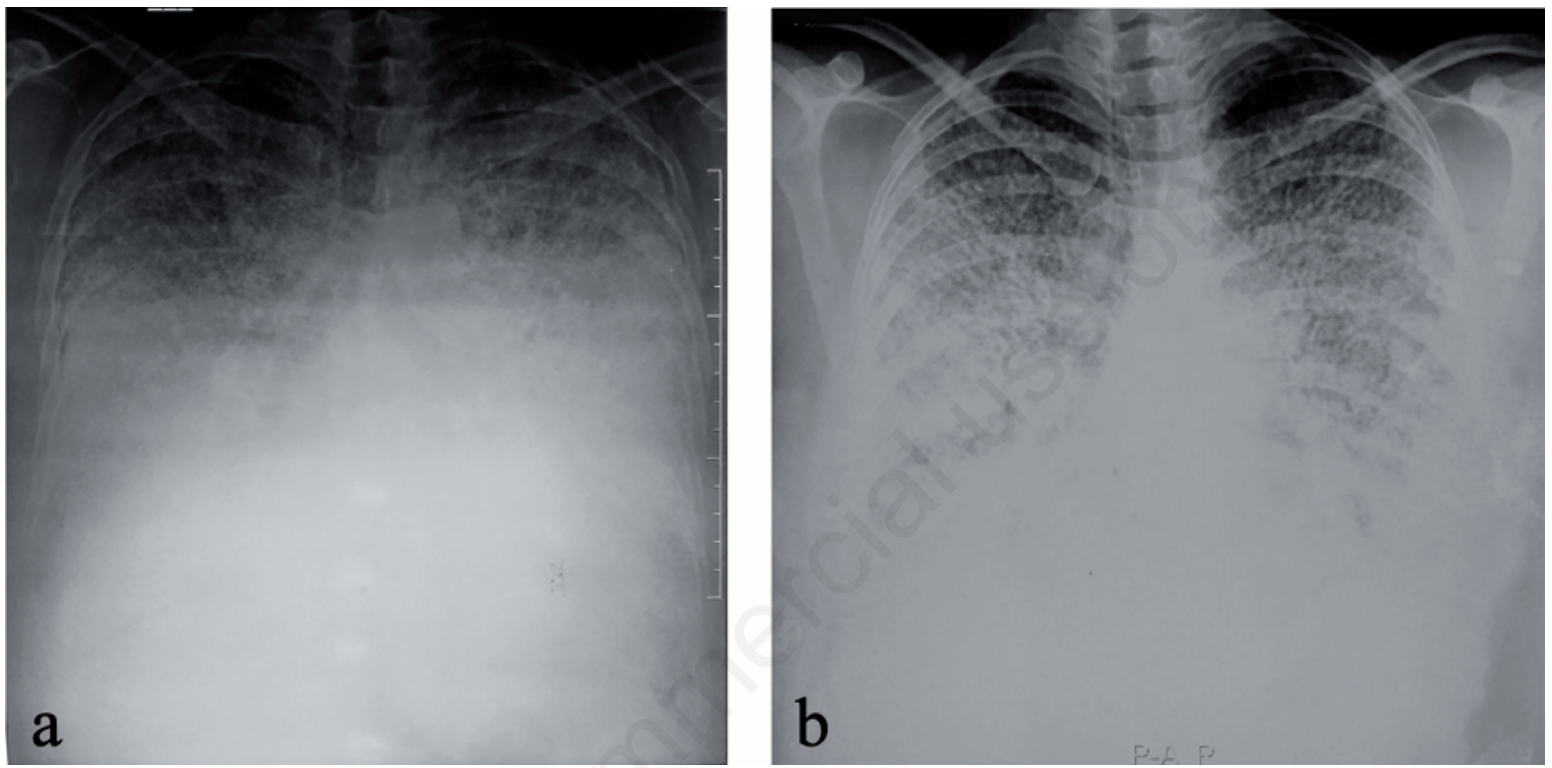

Figure 1. a) Case \# 1: posteroanterior (PA) chest radiograph showing bilateral diffuse, dense, nodular opacities involving predominantly the middle and lower lung zones with obliteration of heart borders and diaphragm. b) Case \#2: posteroanterior (PA) chest radiograph showing diffuse, bilateral micronodular calcifications with sandstorm appearance and fine reticulations, predominantly in the middle and lower zones.
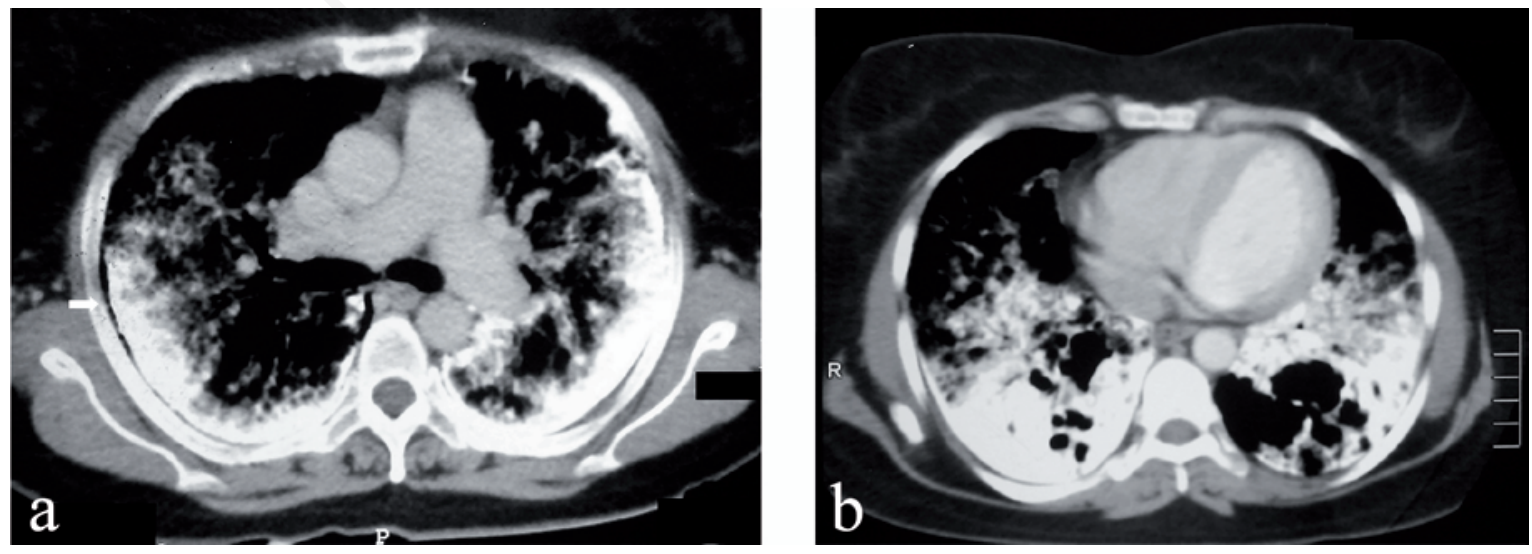

Figure 2. a) Case \#1: mediastinal window of chest high-resolution computed tomography (HRCT) scan showing multiple calcified nodules predominantly in lower lobes and in sub-pleural location with characteristic black pleura sign (arrow). b) Case \#2: mediastinal window of chest high-resolution computed tomography (HRCT) scan showing dense areas of consolidation (calcific agglomerates) located predominantly in subpleural and posterior segments of both the lower lobes. 
ventricle ejection fraction of 58\%. The liver and kidney function test along with the serum electrolytes and parathyroid hormone were normal.

Based on classical HRCT findings accompanied with normal biochemical and other systemic examination, a diagnosis of PAM was made. Patient was not subjected to bronchoscopy in view of hypoxemia and pathognomonic radiological findings that helped in clinching the diagnosis. Her family was screened, and the only brother was found to be in good health with CXR being unremarkable. Patient was started on tab etidronate $(10 \mathrm{mg} / \mathrm{kg}$ per day) and enrolled in the rehabilitation programme. Patient has improved symptomatically after 4 months of treatment, with dyspnea relieved from mMRC grade III to grade I and resting oxygen saturation of $95 \%$. Her follow up chest X-ray did not show any changes as compared to her baseline radiograph.

\section{Case \#2}

A 49-year-old Indian female, never smoker, was referred to the outpatient department for abnormal CXR findings observed incidentally while undergoing pre anaesthesia check-up for paraumbilical hernioplasty. Patient was asymptomatic, denied history of respiratory or constitutional symptoms and was on no medications other than oral hypoglycaemic agents, which she was taking for diabetes mellitus for last 5 years. Physical examination was unremarkable although on auscultation of chest, bilateral inspiratory crackles were heard over all the lung fields. Review of CXR revealed diffuse, bilateral reticulonodular shadows (Figure 1b).

Oxygen saturation $\left(\mathrm{SpO}_{2}\right)$ at room air was $97 \%$. PFT revealed mild restrictive defect with mild reduction in diffusion capacity: $\mathrm{FEV}_{1} 79 \%$, FVC $73 \%$, TLC $71 \%$ and DLCO $66 \%$. Patient was able to cover $460 \mathrm{~m}$ on $6 \mathrm{MWT}$ without significant desaturation (minimum $\mathrm{SpO} 2$ of $95 \%$ ). HRCT chest was done and we observed that there were dense areas of consolidation located predominantly in subpleural and posterior segments of both the lower lobes which were of calcium density (Figure 2b), with crazy paving pattern (areas of ground glass opacity with septal thickening) seen throughout the lung parenchyma (Figure $3 b$ ). Few areas with cystic changes were seen adjacent to areas of consolidation in lower lobes. Echocardiography was normal. Serum calcium and phosphate levels were within normal limits.

In view of the typical above-mentioned radiological findings in an asymptomatic individual, a diagnosis of PAM was made. Patient was kept under observation and 1-year post follow up, she continues to be asymptomatic.

\section{Discussion}

Pulmonary alveolar microlithiasis, also known as Harbitz syndrome (after the scientist who first described it histopathologically), is a rare genetic lung disorder that has a worldwide distribution albeit variable [4]. Although the majority of cases are reported from Asia, particularly from Turkey, China, Japan and India, it is also widely prevalent in Europe (Italy) and USA [1]. There is no obvious gender predilection, however a slight male preponderance was reported in a recent comprehensive review of the disease [1]. In 2015, an all-inclusive review of worldwide prevalence of PAM was reported with 80 cases (out of 1022) from India, after which, to the best of our knowledge, 10 cases have been reported since $[1,2]$.

It is known to have both, familial and sporadic occurrence. Familial cases constitute $35-50 \%$ of all PAM cases and has autosomal recessive inheritance with high penetrance and horizontal pattern thereby commonly seen in children of consanguineous marriages and among siblings [5,6]. Inactivating genetic mutation of SLC34A2 (solute carrier family 34 member 2) gene located on short arm of chromosome 4, accounts for its pathogenesis, which encodes for sodium-dependent phosphate transport protein $2 \mathrm{~b}$, $\mathrm{NaPi}-2 \mathrm{~b}$ [5]. The physiological role of the gene in phosphate transport and clearance from the alveolar spaces is affected by its dysfunction causing phosphate to accumulate in the lumen which then combines with calcium and leads to the formation of microliths knowns as calcospherites.

PAM is presented across all age groups, from premature neonates to octogenarians although it is more frequently seen in
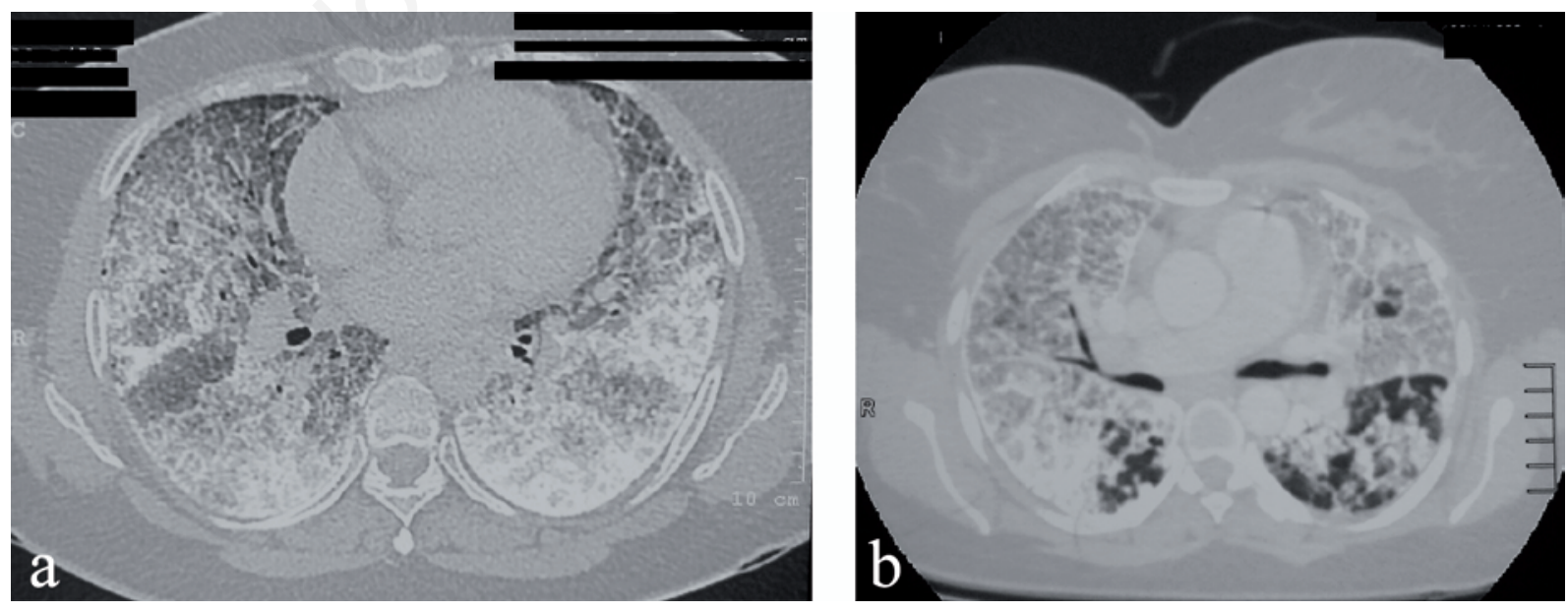

Figure 3. a) Case \#1: high-resolution computed tomography (HRCT) chest axial sections showing ground glass attenuation with inter and intralobular septal thickening with multiple calcified nodules predominantly in lower lobes (posterior segments) and in sub-pleural location. b) High-resolution computed tomography (HRCT) chest axial sections showing diffuse ground-glass attenuation and septal thickening with calcified nodules, demonstrating crazy-paving pattern in both the lungs. 
second and third decades of life [1,5]. The disease has an indolent course with a clinical presentation ranging from asymptomatic (in more than $50 \%$ of patients) in the initial years to frank respiratory failure and cor pulmonale in the later stages. Non-productive cough and exertional dyspnea are the most common symptoms with chest pain, fatigue, hemoptysis, cyanosis, and pneumothorax seen in few. Physical examination commonly reveals finger clubbing in advanced stages. Hallmark of this disorder is the clinicoradiological dissociation where even with vivid radiological findings, patients are either asymptomatic or diagnosed incidentally on screening of the family or due to studies done for another reason [2]. PFTs are used as a monitoring tool as they are normal in the early stages, whereas restrictive pattern with reduction in DLCO is observed later in the course when there is extensive parenchymal involvement. Typically, serum calcium and phosphate are within normal limits [7]. Extrapulmonary calcium deposition has been reported in pleura, diaphragm, lumbar sympathetic chain and in various organs, particularly male genitalia including testicles and seminal vesicles (6-9\% of the male population) [8].

Chest radiograph shows a characteristic snowstorm or sandstorm appearance of diffuse, hyperdense (calcium density), micronodular (nodules with a diameter of $<1 \mathrm{~mm}$ ) shadows predominantly in middle and lower zones with relative sparing of apices [9]. The microliths are typically of uniform size with a distinct border and distributed throughout the lungs, although they tend to be more confluent forming dense consolidations in the medial and inferior regions. Black pleura sign is another classical finding which is due to subpleural cystic changes seen as a linear radiolucency at the pleural boundaries abutting the heart, diaphragm and pleura between ribs and dense pulmonary opacities [10]. Extensive number and density of microliths especially in the medial and inferior regions often causes obscuring of borders of heart and diaphragm known as vanishing heart phenomenon [5]. HRCT reveals widespread micronodular calcifications primarily located along bronchovascular bundles, subpleural and perilobular regions and preferentially deposited in the posterior segments of the lower lobes [11]. Diffuse ground glass opacities with interlobular septal thickening due to accumulation of microliths along the periphery of the secondary pulmonary lobules is often apparent and gives rise to crazy paving appearance. Castellana et al. have described and divided the radiological appearance of PAM into four evolutionary phases i.e. precalcific, typical sandy appearance, obscuring of cardiac borders, and lastly with involvement of pleura [1].

Bronchoalveolar lavage often demonstrates microliths that are spherical ovoid, periodic acid-Schiff-positive and consist of calcareous concentric lamellated, 'onion-skin' appearance around a central nucleus. They are composed of calcium and phosphorus (in ratio of 2:1) [12]. To date, the best diagnostic schedule for PAM is the association of BAL and chest HRCT, but simple chest X-ray may be sufficient in the presence of familial cases. This diagnostic approach allows to limit the biopsy to selected cases.

The dense micronodular pattern observed in PAM can mimic various diseases associated with miliary dissemination such as tuberculosis (TB), fungal infections, sarcoidosis, pulmonary hemosiderosis, pulmonary alveolar proteinosis (PAP), pneumoconiosis including silicosis and amyloidosis. PAM is perhaps most often confused with tuberculosis as regions with high prevalence of TB and PAM frequently overlap as seen in India, China and Turkey [1]. In a review by Ganesan et al., about one third of cases from India were wrongly diagnosed as TB which could be due to both having similar imaging features and lack of knowledge of PAM along with high tendency to suspect TB in an endemic area
[13]. A high index of suspicion is needed for correct diagnosis and to limit the use of unnecessary therapy. The first differentiating feature of PAM from all the earlier-mentioned differentials is that they all have a more severe presentation than PAM. PAM involves the middle and lower lobes with relative sparing of the apices, in contrast to pulmonary tuberculosis. Sputum examination helps in exclusion of TB. In doubtful cases, the search for family cases and the execution of BAL or biopsy can be decisive. Bilateral central and symmetrical lung opacities with relative sparing of apices and costophrenic angles with discrete calcifications visualized within mediastinal window settings distinguish PAM from PAP. Mediastinal adenopathy and parenchymal abnormality with perilymphatic distribution of nodules in sarcoidosis and eggshell calcification with positive history of occupational exposure in pneumoconiosis aids in discriminating them from PAM. Histopathological specimens further helps in distinguishing from infectious aetiologies, amyloidosis (Congo-red positive staining) and hemosiderosis (hemosiderin laden macrophages).

In patients who have diffuse pulmonary calcifications on imaging, the differentials can be broadly divided into two categories, namely, metastatic and dystrophic calcification, wherein calcium deposition occurs in underlying normal (secondary to extrapulmonary condition) or damaged lung tissue, respectively [14]. The causes and differentiating features are briefly described in Table 1.

Till date, there is no established therapy for PAM with treatment largely being supportive, including supplemental oxygen therapy. Limited number of studies have found etidronate to improve lung function and reduce radiographic opacification of the lung [15]. Etidronate inhibits crystal formation and bone mineralization in addition to inhibition of osteoclast driven bone resorption. Systemic corticosteroids and whole lung lavage have been shown to be ineffective [5]. Lung transplantation has proven to be successful in end stage disease with no report of recurrence [16]. Recently, the first case of bilateral lung transplantation performed in India was reported with successful outcome and no recurrence post 1 year of transplant [17]. Patients of PAM have highly variable course of illness with some remaining stable for decades and others developing pulmonary fibrosis. Death often occurs in fifth decade of life primarily due to respiratory failure [1,2].

\section{Conclusions}

PAM is a rare inherited disorder in which patients present with either nonspecific signs and symptoms or are asymptomatic. Clinico-radiological dissociation is a commonly seen feature of the disease. Family screening is an important part of the work up and pathognomonic radiological findings helps to confirm the diagnosis. No specific treatment is present although for symptomatic patients, management depends on severity of the disease.

\section{References}

1. Castellana G, Castellana G, Gentile M, et al. Pulmonary alveolar microlithiasis: Review of the 1022 cases reported worldwide. Eur Respir Rev 2015;24:607-20.

2. Tendolkar M. An "Orphan" in search of a treatment: Pulmonary alveolar microlithiasis. Lung India 2019;36:553.

3. Viswananthan R. Pulmonary alveolar microlithiasis. Thorax 1962;17:251-6. 
4. Harbitz F. Extensive calcification of the lungs as a distinct disease. Arch Intern Med (Chic) 1918;XXI:139-46.

5. Saito A, McCormack FX. Pulmonary alveolar microlithiasis. Clin Chest Med 2016;37:441-8.

6. Castellana G, Gentile M, Castellana R, et al. Pulmonary alveolar microlithiasis: clinical features, evolution of the phenotype, and review of the literature. Am J Med Genet 2002;111:220-4.

7. Tachibana T, Hagiwara K, Johkoh T. Pulmonary alveolar microlithiasis: Review and management. Curr Opin Pulm Med 2009; 15:486-90.

8. Castellana G, Carone D, Castellana M. Microlithiasis of seminal vesicles and severe oligoasthenospermia in pulmonary alveolar microlithiasis (PAM): report of an unusual sporadic case. Int J Fertil Steril 2015;9:137-40.

9. Balikian JP, Fuleihan FJ, Nucho CN. Pulmonary alveolar microlithiasis. Report of five cases with special reference to roentgen manifestations. Am J Roentgenol Radium Ther Nucl Med 1968;103:509-18.

10. Felson B. Thoracic calcifications. Dis Chest 1969;56:330-43.

11. Cluzel P, Grenier P, Bernadac P, et al. Pulmonary alveolar microlithiasis: Ct findings. J Comput Assist Tomog 1991;15: 938-42.

12. Pracyk JB, Simonson SG, Young SL, et al. Composition of lung lavage in pulmonary alveolar microlithiasis. Respiration 1996;63:254-60.

13. Ganesan N, Ambroise MM, Ramdas A, et al. Pulmonary alveolar microlithiasis: an interesting case report with systematic review of Indian literature. Front Med 2015;9:229-38.

14. Belém LC, Zanetti G, Souza AS, et al. Metastatic pulmonary calcification: State-of-the-art review focused on imaging findings. Respir Med 2014;108:668-76.

15. Ozcelik U, Yalcin E, Arıurek M, et al. Long-term results of disodium etidronate treatment in pulmonary alveolar microlithiasis. Pediatr Pulmonol 2010;45:514-7.

16. Stamatis G, Zerkowski H, Doetsch N, et al. Sequential bilateral lung transplantation for pulmonary alveolar microlithiasis. Ann Thorac Surg 1993;56:972-5.

17. Jindal A, Rahulan V, Balasubramani G, et al. Pulmonary alveolar microlithiasis: A rare disease treated with lung transplantation, first case from India. Lung India 2019;36:546. 